\title{
EVALUATION OF THE COORDINATION BETWEEN CHINA'S TECHNOLOGY AND ECONOMY USING A GREY MULTIVARIATE COUPLING MODEL
}

\author{
Qinzi XIAO ${ }^{1,2}$, Miyuan $\mathrm{SHAN}^{1}$, Mingyun $\mathrm{GAO}^{1,3 *}$, Xinping $\mathrm{XIAO}^{4}$, Huan $\mathrm{GUO}^{5}$ \\ ${ }^{1}$ School of Business Administration, Hunan University, Changsha, 410082 Hunan, PR China \\ ${ }^{2}$ Asper School of Business, University of Manitoba, MB R3T 2N2 Winnipeg, Canada \\ ${ }^{3}$ NUS Business School and the Logistics Institute-Asia Pacific, \\ National University of Singapore, S(117592), Singapore \\ ${ }^{4}$ School of Science, Wuhan University of Technology, Wuhan, 430070 Hubei, China \\ ${ }^{5}$ School of Mathematics and Computer Science, Jianghan University, Wuhan, 430056 Hubei, China
}

Received 29 June 2019; accepted 23 August 2020

\begin{abstract}
As extremely complex interactions exist in the process of economic research and development, a novel grey multivariable coupling model called $\operatorname{CFGM}(1, \mathrm{~N})$ is proposed to evaluate the coordination degree between China's technology and economy with limited information. This proposed model improves the aggregation in $\operatorname{GM}(1, \mathrm{~N})$ model through the Choquet integral among $\lambda$-fuzzy measure, which can reflect interactions among factor indexes. Meanwhile, it can estimate the coordinate parameters via the whale optimization algorithm and obtains the coupling coordination degree combining with grey comentropy. To verify the proposed model, a case study using a dataset from China's technology and the economic system is conducted. The CFGM $(1, N)$ model has a better performance in the convergence and interpretability, as compared to the three heuristic algorithm and two classical approaches. Our finding suggests that China's technology and the economic system is still relatively coordinated. Results also reveal that there exists strong negative cooperation between the comprehensive human input and the comprehensive capital investment in this system.
\end{abstract}

Keywords: technology and economic system, Choquet integral, GM(1,N) model, whale optimization algorithm, coordination degree.

JEL Classification: C02, C52, O11.

\section{Introduction}

Technology and economy are the two driving forces of contemporary social progress (Krammer, 2017; Block et al., 2013). Economic growth is accompanied by technological progress; conversely, technological progress accelerates economic growth. Therefore, researching the

*Corresponding author. E-mail: wh14_gao@126.com

Copyright $\odot 2020$ The Author(s). Published by Vilnius Gediminas Technical University

This is an Open Access article distributed under the terms of the Creative Commons Attribution License (http://creativecommons. org/licenses/by/4.0/), which permits unrestricted use, distribution, and reproduction in any medium, provided the original author and source are credited. 
coordination between China's technological capability and economic development promotes the coordinated and sustainable development of both factors; such research also has practical significance for the government to make scientific decisions and perform macroeconomic management (Zhang, 2014).

The coordinated development of technology and economic society is a universal concern (Neokosmidis et al., 2013; Krammer, 2017). The coordination between technology and economy refers to the state and process of mutual support, promotion, and matching between economic development and technological progress (Yuan et al., 2020). Coordination degree is the description of the above state, that is, the degree of matching between economic development and technological progress (Sun, 1996). Pradhan et al. (2014) designed a panel vector autoregressive model to investigate the relationship between development and economic growth in G20 (or Group of Twenty). Ishida (2015) developed two different multivariate models, namely, a five-variable model corresponding to the production model and a four-variable model corresponding to the energy demand model, to handle the influence of Japan's technology investment on economic growth and energy consumption. Hong (2017) examined the Granger causality between research and development (R\&D) investment and economic growth in Korea. Wang and $\mathrm{Xu}$ (2009) used the analytic hierarchy process to comprehensively evaluate the coordinated development of regional technology and economy and showed the degree of coordination between technology and economic development in various regions. Jiang (2011) predicted the future coordinated development of regional technology and economic systems from a dynamic perspective. He established a nonlinear autoregressive model for the coordinated development of regional technology and economic systems and analyzed the dynamic evolution process of the model.

Technology and economic systems are nonlinear complex systems with space-time coupling. The interaction of interdependence and conditionality are present among related variables; at the same time, these related variables affect the changes in system characteristic variables (Coccia, 2014). In the case of GDP (Gross Domestic Product), technological manpower and fund are important factors that influence economic growth. Generally, the more scientific and technological manpower inputs provided, the greater scientific research funds required. The impact of manpower input on industrial output is regulated by funding input, whereas the impact of funding on industrial output depends on the exertion of talent advantage. In addition, technology and economic systems have less data, time lag, and complex mechanism due to the lack of statistical data and low statistical data quality in China. Fu and Zheng (2018) introduced a grey time-delay correlation analysis to improve multi-dimensional grey model GM(1,N) and established a multivariate $\mathrm{GM}(1, \mathrm{~N})$ coordination model. For the system modeling problem with interaction effect, Wang (2017) and Ding et al. (2018) introduced the cross terms of relevant factor sequences into the grey action of the classic $\mathrm{GM}(1, \mathrm{~N})$ model; they also respectively constructed $\mathrm{GM}(1, \mathrm{~N})$ model with interaction effect, which can effectively reflect the interaction effect in multivariate system modeling. However, the functional relationship of driving factor variable may also be nonlinear in practical applications. The Choquet integral is a non-linear model which does not assume the independence of one element from another, and it has been widely applied in the fuzzy multi-attribute group decision-making problems (Aggarwal, 2017; Zhang et al., 2017; Kadaifci et al., 2020). 
The aim of this paper is just to employ a nonadditive technique, the Choquet integral, to aggregate various performance values of $\mathrm{GM}(1, \mathrm{~N})$. A novel grey multivariate coupling model (abbreviated as $\operatorname{CFGM}(1, \mathrm{~N})$ ) is established. The parameter identification and Whale optimization algorithm (WOA) of the model are also investigated. The proposed model can not only eliminate the overlapped information, but can also reflect the interaction among factors set with limited information. Finally, the $\operatorname{CFGM}(1, N)$ model is adopted to the evaluate the coordination between China's S\&T and economy system.

\section{The grey multivariate coupling evaluation model $(\mathrm{CFGM}(1, \mathrm{~N}))$}

To evaluate the coordination of the economy system, a grey multivariate coupling evaluation model (abbreviated as $\operatorname{CFGM}(1, \mathrm{~N})$ ) is established in this section. The proposed model can reflect the interaction in the classical $\operatorname{GM}(1, \mathrm{~N})$ model (see Subsection 2.1) inspired by the Choquet integral among $\lambda$-fuzzy measure (see Subsection 2.2). And the theoretical discussion and the grey comentropy are displayed in Subsection 2.3.

\section{1. $\operatorname{GM}(1, N)$ model}

The $\operatorname{GM}(1, N)$ model is a classic grey model, which can evaluate the relationship between the behavior sequence and the factors sequence. Let the behavior sequence be $x_{1}^{(0)}=\left(x_{1}^{(0)}(1), x_{1}^{(0)}(2), \cdots, x_{1}^{(0)}(n)\right)$, and the factor sequences of system be

$$
\begin{gathered}
x_{2}^{(0)}=\left(x_{2}^{(0)}(1), x_{2}^{(0)}(2), \cdots, x_{2}^{(0)}(n)\right) \\
x_{3}^{(0)}=\left(x_{3}^{(0)}(1), x_{3}^{(0)}(2), \cdots, x_{3}^{(0)}(n)\right), \\
\vdots \\
x_{N}^{(0)}=\left(x_{N}^{(0)}(1), x_{N}^{(0)}(2), \cdots, x_{N}^{(0)}(n)\right)
\end{gathered}
$$

then the definition of $\operatorname{GM}(1, N)$ is given as follows.

Definition 1. The following Eq. (1) is named $\mathrm{GM}(1, \mathrm{~N})$ model, that is,

$$
x_{1}^{(0)}(k)+a z_{1}^{(1)}(k)=\sum_{i=2}^{n} b_{i} x_{i}^{(1)}(k)
$$

where $x_{i}^{(1)}(k)=\sum_{j=1}^{k} x_{i}^{(1)}(j)$ is the 1 -st accumulated generation about $x_{i}^{(0)}, i=1,2, \cdots, N$, and $z_{1}^{(1)}(k)$ is the background value of the grey derivative sequence, namely, $z_{1}^{(1)}(k)=\frac{1}{2}\left(x_{1}^{(1)}(k)+\right.$ $\left.x_{1}^{(1)}(k-1)\right)$.

From a perspective of system control, the GM(1,N) model is an analysis model or factor model, it does not has comprehensive information and is generally not suitable for forecasting (Deng, 2002). The parameters $a$ is called development coefficient, and $b_{i}$ is named as coordination coefficient about $x_{i}$. The values of $a$ and $b_{i}$ can be used to make an evaluation about the observation system. 
From a perspective of social resources, $x_{1}^{(0)}(k)$ is the system behavior, $x_{1}^{(0)}(k)+a z_{1}^{(1)}(k)$ is the function of the behavior sequence (Xiao et al., 2020d). $x_{i}^{(1)}(k)$ is the behavior of resources $i$, where $i=2,3, \cdots, N$. And $\sum_{i=2}^{N} b_{i} x_{i}^{(1)}(k)$ is treated as the resource-combination, which is an linear aggregation about the factor sequences of system at the moment $k$. It is worth noting that these behavior resources are assumed to be mutually independent in this aggregation. The linear aggregation may not evaluate the behavior system reasonably when the system is affected by many factors having interaction effects additivity. Then, this paper improves the aggregation in $\mathrm{GM}(1, \mathrm{~N})$ model through the Choquet integral.

\subsection{Fuzzy measures and Choquet integral}

Since fuzzy measures may be used as an effective method to express the interactions among behavior resources, $\lambda$-fuzzy measure is easy to calculate among all fuzzy measures, and fuzzy integral is able to perform aggregation of criteria in some decision making problems (Marichal, 2004). Thus, in this section, the definitions of fuzzy measure, $\lambda$-fuzzy measure, and the Choquet integral are presented as follows.

Definition 2. Let $x_{1}^{(0)}$ be the dependent sequence, $X=\left\{x_{2}^{(0)}, x_{3}^{(0)}, \cdots, x_{N}^{(0)}\right\}$ be a finite set of factors, $P(X)$ be a power set of $X,(X, P(X))$ be a measurable space. A fuzzy measure $\mu$ on $X$ is a set function $\mu: P(X) \rightarrow[0,1]$, satisfying the following conditions (Sugeno, 1974).

(1) $\mu(\varnothing)=0, \mu(X)=1$;

(2) $\forall A, B \in P(X)$, if $A \subseteq B$, then $\mu(A) \leq \mu(B)$.

The common fuzzy measures have many types of particular construct, such as possibility measures, necessity measures, belief measures, k-addictive fuzzy-measures and so on (Meng et al., 2015). Since these fuzzy-measures need a lot of raw data, the following $\lambda$-fuzzy measure is usually used instead of common fuzzy measures because it can reduce the difficulty of data collection (Chiang, 1999):

$$
\mu(A \bigcup B)=\mu(A)+\mu(B)+\lambda \mu(A) \mu(B) .
$$

$\forall A, B \in P(X), A \cap B=\varnothing$, and $\lambda \geq-1$. The parameter $\lambda$ determines interaction between the attributes. If $\lambda=0, \lambda$-fuzzy measure reduces to simply an additive measure, and all attributes are independent of each other; if $-1<\lambda<0$, it is a sub-additive measures, and negative cooperation exists among all attributes; if $\lambda>0$, there are super-additive measures and active cooperation among all attributes.

When $\lambda$ is known, then the fuzzy measure function about the attribute set $A$, denoted by $\mu(A)$, can be expressed through the $\phi_{s}$ transfer function in the following (Takahagi, 2008).

Definition 3. Assuming $\phi_{s}:[0,1] \times[0,1] \rightarrow[0,1]$ satisfies Eq. (2), then $\phi_{s}$ is defined as a transfer function about $\lambda$ fuzzy measure. Using the attribute weights $w_{i}, i=1,2, \cdots, N-1$ and the transfer function $\phi_{s}$ to determine the fuzzy measure, then the fuzzy measure, about $A$, obtain $\mu(A)$ : 


$$
\begin{gathered}
\phi_{s}(\xi, w)=\left\{\begin{array}{rr}
0, & \xi=1, w=0 \\
1, & \xi=0, w=1 \\
0, & \xi=0, w<1 \\
\frac{\left((1-\xi)^{2} / \xi^{2}\right)^{w}-1}{\left((1-\xi)^{2} / \xi^{2}\right)-1}, \text { otherwise }
\end{array}\right. \\
\mu(A)=\phi_{s}\left(\xi, \sum_{u_{j} \in A} w_{j}\right), \quad \forall A \in P(X),
\end{gathered}
$$

where $\xi=1 /(\sqrt{1+\lambda}+1)$.

Since the Choquet integral is able to perform aggregation of criteria even when mutual preferential independence is violated, it is widely applied to comprehensive evaluation $(\mathrm{Qu}$ et al., 2017; Feng et al., 2018).

Definition 4. The discrete Choquet integral of set function $f: X \rightarrow[0,1]$ with respect to fuzzy measure $\mu$ is defined as follows (Grabisch, 1996):

$$
\int f d \mu=\sum_{i=1}^{N-1}\left[f\left(x_{(i)}\right)-f\left(x_{(i-1)}\right)\right] \mu\left(A_{(i)}\right)
$$

where $(i)$ indicates a permutation on $X$ such that

$$
\left.f\left(x_{(1)}\right) \leq f\left(x_{(2)}\right)\right) \leq \cdots \leq f\left(x_{(N-1)}\right), f\left(x_{(0)}\right)=0 \text {, and } A_{(i)}=\left\{x_{(i)}, x_{(i+1)}, \cdots, x_{(N-1)}\right\} .
$$

Thus defined, the Choquet integral coincides with the weighted arithmetic sum as soon as the fuzzy measure $\mu\left(A_{(i)}\right)$ is additive, whose weight depends on the permutation of $f\left(x_{(i)}\right)$. As a consequence, only the Choquet integral with respect to an additive measure is decomposable.

In order to address some multicriteria decision making problems with a large number of interactive indices, many scholars introduced the Choquet integral into the traditional grey relational analysis (GRA) to obtain novel GRA models, such as Fuzzy Grey Choquet Integral (Tian et al., 2019), Choquet integral-based GRA (Hu, 2008), Interval-valued Pythagorean Fuzzy GRA (Khan \& Abdullah, 2018), and Grey Information Coverage Interaction Relational Degree (Xiao et al., 2020a). This paper will combine the Choquet integral with $\operatorname{GM}(1, N)$ to establish novel grey multivariable coupling model $(\mathrm{CFGM}(1, \mathrm{~N}))$.

\section{3. $\operatorname{CFGM}(1, N)$ model}

In traditional GM(1,N) model, $F_{k}=\sum_{i=2}^{N} b_{i} x_{i}^{(1)}(k)$ is the resource-combination, which only aggregates these factors independently but ignores the interactions among factors. For these $N-1$ factors, there may be as many as $2^{N-1}$ interaction effect from different factor subsets. Meanwhile, for the factor $x_{i}$, let $f_{k}\left(x_{i}\right)=\mathrm{v}_{i} x_{i}^{(1)}(k)$ be an observation at the moment $k$, where $i=2,3, \cdots, N, k=2,3, \cdots, n$, and $v_{i}$ is an accommodation coefficient which can ensure $\mathrm{v}_{i} x_{i}^{(1)}(k) \in[0,1]$. In comparison with the traditional $\mathrm{GM}(1, \mathrm{~N})$ model with additivity assumption, a non-additive resource-combination $F_{k}$ combined with the Choquet integral is formulated as 


$$
F_{k}=\int f_{k} d \mu=\sum_{i=1}^{N-1}\left[f_{k}\left(x_{(i)}\right)-f_{k}\left(x_{(i-1)}\right)\right] \mu\left(\left\{x_{(i)}, x_{(i+1)}, \cdots, x_{(N-1)}\right\}\right),
$$

where $0=f_{0}\left(x_{(0)}\right) \leq f_{k}\left(x_{(1)}\right) \leq f_{k}\left(x_{(2)}\right) \leq \cdots \leq f_{k}\left(x_{(N-1)}\right)$, and $\mu\left(\left\{x_{(i)}, x_{(i+1)}, \cdots, x_{(N-1)}\right\}\right)$ means the fuzzy measure of the factor set $\left\{x_{(i)}, x_{(i+1)}, \cdots, x_{(N-1)}\right\}$.

Definition 5. The Choquet fuzzy GM(1,N) model, namely $\operatorname{CFGM}(1, N)$, is formulated as

$$
x_{1}^{(0)}(k)+a z_{1}^{(1)}(k)=b \int f_{k} d \mu .
$$

Corresponding with the $\mathrm{GM}(1, \mathrm{~N})$ model, the coordination coefficient $b_{i}$ is expressed as

$$
b_{i}=b v_{i} .
$$

The development coefficient $a$ reveals the development of systems. When $a<0$, it means that $\sum_{j=1}^{k-1} x_{i}^{(1)}(j)$, the accumulated value of the front $k=1$ behavior $x_{i}$, always has played a positive role on the behavior at any time $k$, which reveals that this system has a certain development ability. Meanwhile, the observation system keeps the quantity coordination. When $a \geq 0$, it means that $\sum_{j=1}^{k-1} x_{i}^{(1)}(j)$ always has played a negative role on the behavior at any time $k$, which reveals this system has no self-development ability.

The coefficient $b_{i}$ can measure the coordination between the behavior and the factor $x_{i}$, $i=2,3, \cdots, N$. When $b_{i}>0$, it reveals that the factor $x_{i}$ has promoted the behavior $x_{1}$, and the observation system keeps the structure coordination. Otherwise, the factor $x_{i}$ has restrained the behavior $x_{i}$.

From Definition 5, GM $(1, \mathrm{~N})$ model (in Definition 1) can be regarded as the special forms of $\operatorname{CFGM}(1, \mathrm{~N})$ model where $\lambda=0$. Namely, the $\operatorname{CFGM}(1, \mathrm{~N})$ model is an expansion of classic $\mathrm{GM}(1, \mathrm{~N})$ under the perspective of the interactive factors.

It is worth noting that this study employs $\lambda$-fuzzy measure and applies to the Choquet integral, the reasons are that $\lambda$-fuzzy measure is easy to calculate and does not need a lot of raw data, which conforms to the poor information characteristics of grey system. However, $\lambda$-fuzzy measure can only express one kind of interaction in one group of indices, it is very important to construct a corresponding appropriate evaluation index system in order to overcome the limitations of $\lambda$-fuzzy measure. There are some possible methods, for example, using hierarchy and grouping indices can divide the index system into several levels that have a kind of interaction (Tian et al., 2019), or reducing the dimensionality of the index system by using principal component analysis and grey relational analysis (see Section 4).

Definition 6. The following Eq. (8) is the $\operatorname{CFGM}(1, N)$ whitening differential equation:

$$
\frac{d x^{(1)}(t)}{d t}+a x_{1}^{(1)}(t)=b\left\{\int f_{t} d \mu\right\} .
$$

As the parameters $v_{3}, v_{4}, \cdots, v_{N}$ are known, then parameter sequence $\hat{P}=(a, b)^{T}$ can be estimated as displayed in Theorem 2 . 
Theorem 1. The least squares estimation of parameter sequence in $\operatorname{CFGM}(1, \mathrm{~N}), \hat{P}=\left(a, b_{2}\right)^{T}$, satisfies Eq. (9):

$$
\hat{P}=\left(B^{T} B\right)^{-1} B^{T} Y,
$$

where $B=\left[\begin{array}{cc}-z_{1}^{(1)}(2) & \int f_{2} d \mu \\ -z_{1}^{(1)}(3) & \int f_{3} d \mu \\ \vdots & \vdots \\ -z_{1}^{(1)}(n) & \int f_{n} d \mu\end{array}\right], Y=\left[\begin{array}{c}x_{1}^{(0)}(2) \\ x_{1}^{(0)}(3) \\ \vdots \\ x_{1}^{(0)}(n)\end{array}\right]$

Proof. It is Obvious.

According the parameter estimation about $(a, b)$, then we can solve the time response function through Theorem 2 .

Theorem 2. If the initial value $x^{(1)}(1)=x^{(0)}(1)$ is given, then the solution of the $\operatorname{CFGM}(1, \mathrm{~N})$ whitening differential equation will satisfy

$$
\hat{x}_{1}^{(1)}=e^{-a t}\left\{b \int_{1}^{t} F(\tau) e^{a_{1} \tau} d \tau+e^{a} x_{1}^{(0)}(1)\right\},
$$

where $F(\tau)$ represents the Choquet fuzzy measure at time $\tau$ as follows:

$$
F(\tau)=\int f_{\tau} d \mu
$$

Proof. The differential equation is $\frac{d x_{1}^{(1)}(t)}{d t}+a x_{1}^{(1)}(t)=b F(t)$. Let $x_{1}^{(1)}(t)=u(t) e^{-a t}$, then $\frac{d x_{1}^{(1)}(t)}{d t}=\frac{d u(t)}{d t} e^{-a t}-a u(t) e^{-a t}$. Hence, $\frac{d u(t)}{d t}=b F(t) e^{a t}, \quad$ and $\int_{1}^{t} \frac{d u(\tau)}{d \tau}=b \int_{1}^{t} F(\tau) e^{a \tau} d \tau$. Moreover, $u(t)=b \int_{1}^{t} F(\tau) e^{a_{1} \tau} d \tau+u(1)$.

Now, Eq. (10) is completely proven.

In Eq. (10), the continuous measure function $F(\tau)$ is unknown but only can be described with a series of discrete observation. Thus, the $\int_{1}^{k} F(\tau) e^{a_{1} \tau} d \tau$ can be constructed with the numerical integration as follows:

$$
\int_{1}^{k} F(\tau) e^{a_{1} \tau} d \tau=\sum_{j=1}^{k} A_{j} F(j) e^{a j}
$$

where $A_{j}$ represents the integral coefficient, and $F(j)$ represents the integral node.

$$
F(j)=\sum_{i=1}^{N-1}\left[f_{j}\left(x_{(i)}\right)-f_{j}\left(x_{(i-1)}\right)\right] \mu\left(\left\{x_{(i)}, x_{(i+1)}, \cdots, x_{(N-1)}\right\}\right) .
$$

For the design of the integral coefficient, refer to the high-precision Newton-Cotes formula as follows:

$$
A_{j}=(k-1) C_{j}^{(k)},
$$

where $C_{j}^{(k)}$ is named as Cotes coefficient. In summary, the solution of Eq. (10) can be expressed as 


$$
\hat{x}_{1}^{(1)}(k)=e^{-a k}\left\{b(k-1) \sum_{j=1}^{k} C_{j}^{(k)} F(j) e^{a j}+e^{a} x_{1}^{(0)}(1)\right\} .
$$

It is worth noting that when $F(t)$ does not significantly change with time, then Eq. (10) can be expressed as

$$
\hat{x}_{1}^{(1)}(k)=e^{-a k}\left\{\frac{b F(k)}{a} e^{-a(k-1)}+e^{a} x_{1}^{(0)}(1)\right\} .
$$

Then, the prediction about the behavior can be gained from the model prediction value $\hat{x}_{1}^{(1)}(k)$ by Eq. (16):

$$
\hat{x}^{(0)}(k)=\hat{x}^{(1)}(k)-\hat{x}^{(1)}(k-1) .
$$

After obtaining the parameters $b$ and $v_{i}$ of the $\operatorname{CFGM}(1, N)$ model, the coordination coefficient $b_{i}(i=2,3, \cdots, N)$ of the $\operatorname{CFGM}(1, N)$ model can be calculated. If the behavior and factors are time series, then $b_{i}$ and $a$ can be obtained by compartmental modeling when $k=N+3, N+4, \cdots, n$, which are respectively called as $b_{i}(k)$ and $a(k)$. Finally, we can define the coordination degree by grey comentropy.

Definition 7. When $B_{i}(k)=\left\{b_{2}(k), b_{3}(k), \cdots, b_{n}(k)\right\}$, the mapping $B_{i} \rightarrow P_{i}$ is the coordinated development coefficient distribution map, and the mapping value is the density value of the distribution.

$$
S(k)=-\sum_{i=2}^{N} p_{i}(k) \ln p_{i}(k)
$$

is the grey comentropy of the dissipative structure, and

$$
p_{i}(k)=\left|b_{i}(k)\right| / \sum_{i=2}^{N}\left|b_{i}(k)\right|, p_{i}(k) \in P_{i},
$$

where $i=2,3, \cdots N ; k=N+3, N+4, \cdots, n$. $S(k)$ describes the coupling coordination degree between behavior and factors in system.

Combined with the comentropy representation of the dissipative structure (Wang et al., 2019), the change of $S(k)$ is used to discriminate the synergistic and ordered degree of the science-economic system

$$
\Delta S(k+1)=S(k+1)-S(k),
$$

where $\Delta S$ represents the comentropy change value of the system from the $k$ period (initial state) to the $k+1$ period (final state). If $\Delta S(k+1)>0$, then the system comentropy is positive, the $\mathrm{S} \& \mathrm{~T}$ and economic system is disordered state, and technology has no positive impact on the economy. If $\Delta S(k+1)<0$, then the system comentropy is negative, the ordered degree of S\&T and economic system gradually increases, and technology has a positive impact on the economy. If $\Delta S=0$, then $S \& T$ and economic system do not produce comentropy change within a certain period, and technology has no impact on the economy. 


\section{Parameter optimization of $\mathrm{CFGM}(1, \mathrm{~N})$ model}

In the previous section, the modeling process of $\operatorname{CFGM}(1, N)$ model, which includes the solution of development coefficient and the main coordination coefficient, is discussed. In this section, the calculation of two important parameters, namely, the interaction coefficient $\lambda$ and the solution of auxiliary parameter $v=\left\{v_{2}, v_{3}, \cdots, v_{N}\right\}$ in observation function $f_{k}$ are discussed.

\subsection{Parameter optimization model}

The mean absolute percentage error (MAPE) of the model is a widely used evaluation metric for modeling (Xiao et al., 2020b). Therefore, $\lambda$ and $v^{*}=\left\{v_{2}, v_{3}, \cdots, v_{N}\right\}$, which can make the MAPE minimum, are selected as the optimal parameters of the model. In addition, the minimum MAPE value is used as the optimization target, and the optimization model of MAPE is established for $r$ and $m$. The objective function is expressed as follows:

$$
\begin{array}{ll}
\operatorname{MAPE}\left(\lambda, \cup_{2}, \cup_{3}, \cdots, \cup_{N}\right)=\frac{1}{n-1} \sum_{k=2}^{n} \frac{\left|\hat{x}^{(0)}(k)-x^{(0)}(k)\right|}{x^{(0)}(k)} \times 100 \% \\
\text { s.t. } \quad \hat{x}^{(1)}(k)=e^{-a k}\left\{b_{2}(k-1) \sum_{j=1}^{k} C_{j}^{(k)} F(j) e^{a j}+e^{a} x_{1}^{(0)}(1)\right\} \\
\quad \hat{x}^{(0)}(k)=\hat{x}^{(1)}(k)-\hat{x}^{(1)}(k-1) \\
\quad f_{k}\left(x_{i}\right)=v_{i} x_{i}^{(1)}(k)
\end{array}
$$

The above optimization model includes measure and absolute value operations. Therefore, effectively solving the model using the traditional optimization method is difficult (Xiao et al., 2020c; Xiao \& Duan, 2020). This study imitates the humpback whale predation process, designs the whale optimization algorithm, and finally solves the model parameters on the basis of the optimization goal. The specific solution process is explained in Subsection 3.2.

\subsection{Whale optimization algorithm}

Whale optimization algorithm (WOA) is a new intelligent optimization algorithm based on population heuristic that is proposed by mimicking the social behavior of humpback whales (Mirjalili \& Lewis, 2016). The hunting mechanism can be simply expressed. After humpback whales search for prey, they initially spiral around the water, gradually narrow their range, and swim to their prey. When the range is determined, they finally capture the prey by using a square or circular bubble attack method. The prey is equivalent to the optimal solution of optimization problem. The search process of humpback whales is equivalent to the searching for solution optimization. The parameters to be optimized $\lambda$ and $\left\{v_{2}, v_{3}, \cdots, v_{N}\right\}$ in the $\operatorname{CFGM}(1, \mathrm{~N})$ are regarded as whale predator target coordinate $X$, which is expressed as follows:

$$
X=\left(\lambda, v_{2}, v_{3}, \cdots, v_{N}\right) .
$$

A whale group consisting of $N_{w}$ humpback whales is constructed. $T_{\max }$ predation operations are then performed. Finally, the optimal predation point coordinates are obtained as 
the optimal solution for the model. Based on the $t$-th $(t=1,2, \cdots, T)$ iteration, in the first $t$-th predation, the optimal predation coordinate of the whale group is $X_{t}^{*}$, which is the value of $\left(\lambda, v_{2}, v_{3}, \cdots, v_{N}\right)$ corresponding to the smallest MAPE value. The whale $k\left(k=1,2, \cdots, N_{w}\right)$ takes one of the three predation strategies, which are encircling, bubble-net attacking, and searching for prey. Its own predator coordinate $X_{t+1, k}$ is updated in the next stage.

WOA starts with a set of random solutions. During the iterative process, the position is updated by randomly selecting whale individuals or the current optimal individual. The different values of parameter $\vec{a}$ control the exploration and mining of WOA. Meanwhile, parameter $p$ affects the exploration or the selective jump execution of the mining process through the parallel iterative optimization of the whales during exploration and mining. In this manner, the whales gradually approach their prey and finally determine the optimal solution of the problem as shown in the Figure 1.

The optimization model in Subsection 3.1 can be established by using WOA, and then model evaluation parameters $\lambda, v_{2}, v_{3}, \cdots, v_{N}$ can be determined. In summary, the specific process of $\operatorname{CFGM}(1, \mathrm{~N})$ evaluation model is illustrated in Figure 2.

As shown in Figure 2, the entire economic coordination evaluation model is divided into seven steps.

Step 1. Data are collected. Research behavior sequence $x_{1}^{(0)}$ and the factor sequences $x_{2}^{(0)}, x_{3}^{(0)}, \cdots, x_{N}^{(0)}$ are determined for subsequent modeling.

Step 2. Accumulate generation is conducted for the behavior and factor sequences. Finally, $x_{1}^{(1)}$ and $x_{2}^{(1)}, x_{3}^{(1)}, \cdots, x_{N}^{(1)}$ are obtained. The mean value generation is also performed after obtaining behavior accumulation sequence $x_{1}^{(1)}$ and $z_{1}^{(1)}$.

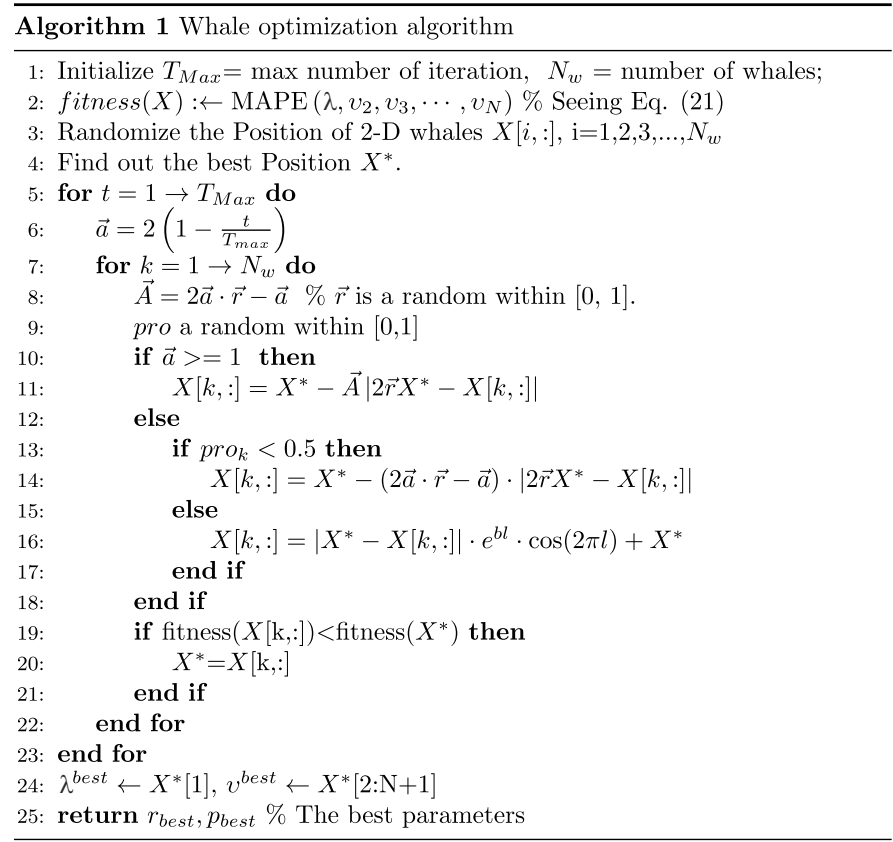

Figure 1. The pseudocode of WOA 


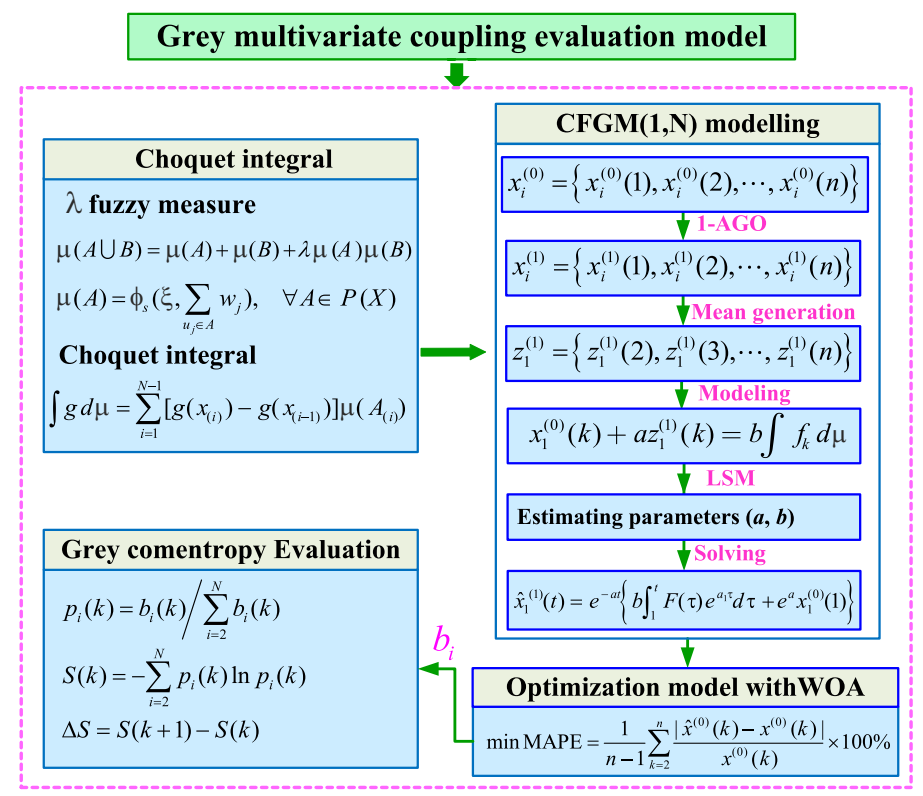

Figure 2. Flow chart of $\operatorname{CFGM}(1, \mathrm{~N})$ evaluation model

Step 3. Fuzzy integral is integrally calculated. The fuzzy measure set is calculated on the basis of interaction parameter $\lambda$. Fuzzy integral $\int f_{t} d \mu$ is then completed.

Step 4. Model parameters $(a, b)$ are estimated by using the least squares method, and MAPE is calculated.

Step 5. Coordination coefficients $\left(b_{3}, \cdots, b_{N}\right)$ are calculated. The parameter sequence $\lambda, v_{3}, v_{4}, \cdots, v_{N}$ of the subsequent modeling is determined by using WOA and MAPE parameter optimization model. Finally, $b_{i}=b v_{i}$, where $i=2,3, \cdots, N$.

Step 6. The dissipative structure grey comentropy is constructed on the basis of the distribution map of coordinated development coefficients $\left(a, b_{2}, b_{3}, \cdots, b_{N}\right)$. The coordination degree is calculated and evaluated.

\section{Case study}

\subsection{Data collection}

To evaluate the coordination degree between China's technology and economy, we obtained the research data (listed in Table 1) from the National Bureau of Statistics of China (2019). As displayed in Table 1, our behavior variable is the Profits of Enterprises (signed as $u_{0}$, unit: 100 million yuan), and basic factors set include $u_{1}, u_{2}, \cdots, u_{5}$, where $u_{1}$ is the Full-time Equivalent of R\&D Personnel (uint: 10,000 man-year), $u_{2}$ is the Expenditure on R\&D (unit: 100 million yuan), $u_{3}$ is the Expenditure on New Products Development (unit: 100 million yuan), $u_{4}$ is the Patent Applications (unit: piece), and $u_{5}$ is the Investment in Fixed Assets (unit: 100 million yuan). 
Prior to the establishment of evaluation model, an interaction testing of the basic factors set are performed as presented in Tables 2. Obviously, there is a strong interaction among the variable systems according to the interaction test in Table 2.

Considering the differences existing in the order of magnitude and the base unit about these basic factors set, the standardization method is adopt as displayed in Eq. (22):

$$
U_{i}=u_{i} / \operatorname{std}\left(u_{i}\right), i=1,2, \cdots, 5,
$$

where $\operatorname{std}\left(u_{i}\right)$ is the standard deviation of $u_{i}$.

Through the Eq. (22), on the one hand, the differences in the order of magnitude and the base unit may have little influence on the following data pre-processing. On the other hand, the standard deviation and variance of these basic factors are unified to $\mathbf{1}$, which can simplify subsequent calculations. To reduce the dimensionality with the principal component analysis in MATLAB software, the first two principal components are selected for subsequent modeling analysis. The descriptions of the two main components are presented in Table 3.

Table 1. Statistics of China's high-tech industry

\begin{tabular}{|c|c|c|c|c|c|c|}
\hline Year & $u_{0}$ & $u_{1}$ & $u_{2}$ & $u_{3}$ & $u_{4}$ & $u_{5}$ \\
\hline 2008 & 2725.1 & 28.50 & 655.20 & 798.40 & 39656 & 4169.20 \\
\hline 2009 & 3278.5 & 32.00 & 774.00 & 925.10 & 51513 & 4882.20 \\
\hline 2010 & 4879.7 & 39.91 & 967.83 & 1006.94 & 59683 & 6944.70 \\
\hline 2011 & 5244.9 & 42.70 & 1237.80 & 1528.00 & 77725 & 9468.46 \\
\hline 2012 & 6186.3 & 52.60 & 1491.49 & 1827.48 & 97200 & 12932.70 \\
\hline 2013 & 7233.7 & 55.90 & 1734.40 & 2069.50 & 102532 & 15557.70 \\
\hline 2014 & 8095.2 & 57.30 & 1922.20 & 2350.60 & 120077 & 17451.72 \\
\hline 2015 & 8986.3 & 59.00 & 2219.70 & 2574.60 & 114562 & 19950.65 \\
\hline 2016 & 10301.8 & 58.02 & 2437.61 & 3000.36 & 131680 & 22786.67 \\
\hline 2017 & 11295.9 & 59.03 & 2644.65 & 3421.30 & 158354 & 26186.55 \\
\hline
\end{tabular}

Table 2. Interaction test of indicator system

\begin{tabular}{|l|c|c|c|c|}
\hline \multicolumn{1}{|c|}{ Source } & SS & MS & F-value & p-value \\
\hline Year & $6.73 \mathrm{E}+10$ & $1.68 \mathrm{E}+10$ & 663.3886 & $5.65 \mathrm{E}-25$ \\
\hline Index & $3.82 \mathrm{E}+09$ & $9.56 \mathrm{E}+08$ & 37.66193 & $3.06 \mathrm{E}-10$ \\
\hline Interaction & $9.01 \mathrm{E}+09$ & $5.63 \mathrm{E}+08$ & 22.19504 & $6.79 \mathrm{E}-11$ \\
\hline SS & $6.34 \mathrm{E}+08$ & 25377461 & & \\
\hline Total & $8.08 \mathrm{E}+10$ & & & \\
\hline
\end{tabular}

Note: SS means Sum of Square; MS means Square of Mean.

Table 3. The loading matrix and the contribution rate of PCA

\begin{tabular}{|c|c|c|c|c|c|c|c|}
\hline Factor & $U_{1}$ & $U_{2}$ & $U_{3}$ & $U_{4}$ & $U_{5}$ & PCV & PVE \\
\hline$x_{2}^{(0)}$ & 0.4319 & 0.4517 & 0.4503 & 0.4502 & 0.4516 & 4.8587 & $97.17 \%$ \\
\hline$x_{3}^{(0)}$ & 0.8874 & -0.1645 & -0.3428 & -0.1032 & -0.2394 & 0.1187 & $2.37 \%$ \\
\hline
\end{tabular}

Note: PCV is principal component variances, that is, the eigenvalues of the covariance matrix of factors. $\mathrm{PVE}$ is the percentage of the total variance explained by each principal component. 
Table 3 shows that the entire basic factors set can be taken placed with these two principal components. The percentage of the total variance of the first principal component reaches up to $97.17 \%$. Considering that the coefficients of the five basic factors on this principal component are all in proximity with each other because their values are approximately 0.45 , then the first principal component can be named comprehensive capital investment. This component is used as the first factor (signed as $x_{2}^{(0)}$ ) for subsequent modeling. As for the second principal component, its PVE makes up $2.37 \%$ of the total variance. Considering that the second principal component accounts for a large proportion on factor $u_{1}$ (reaching 0.8874), it can be called comprehensive human input. Then this component is used as the second factor variable (signed as $x_{3}^{(0)}$ ) for subsequent modeling. The rest three principal components are ignoring, because the cumulative variance of them is only $0.46 \%$.

In addition, original data $u_{0}$ are standardized according to Eq. (22), namely, $x_{1}^{(0)}=u_{0} / \operatorname{std}\left(u_{0}\right)$. Then this sequence data is used as the behavior for this evaluation system. The behavior sequence and the above two principal components are made up of the modeling dataset for subsequent analyzing, which are displayed in Figure 3.

Figure 3 shows that the behavior of system, $x_{1}^{(0)}$ (approximate range is from 1 to 4 ), factors set $x_{2}^{(0)}$ (approximate range is from 2 to 10), and $x_{3}^{(0)}$ (approximate range is from 1 to 2 ) are all stable, which means they all meet the conditions of grey modeling.

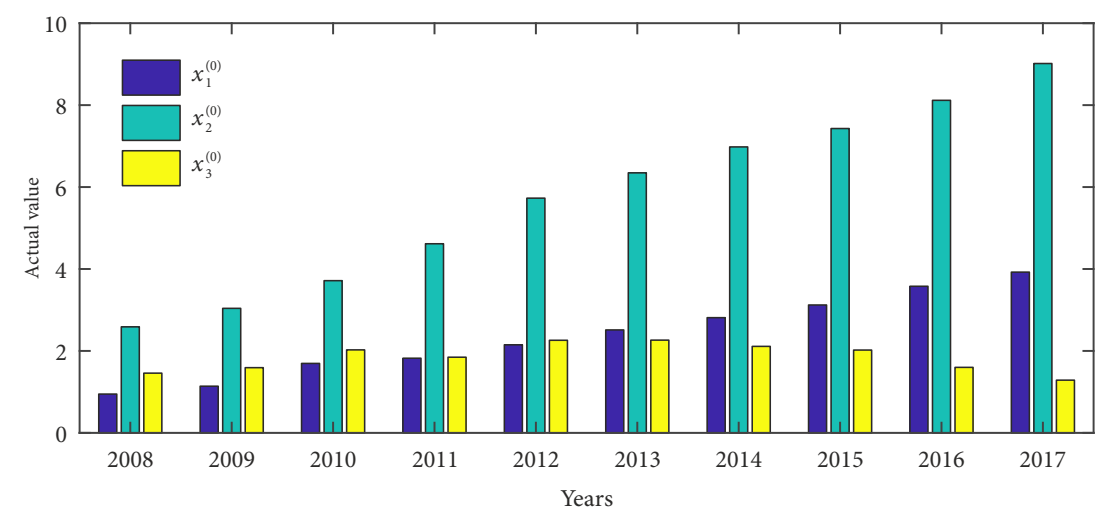

Figure 3. The description about modeling dataset

\subsection{Model comparison}

In this subsection, two comparison research have been done. The one is to evaluate the performance of WOA in $\operatorname{CFGM}(1, N)$ model, the other is to evaluate the model interpretation of $\operatorname{CFGM}(1, N)$ with two traditional grey multivariate models. And four common accuracy criteria are used to validate the effectiveness of the models as follows:

$$
\text { Avg-Step }=\frac{1}{M} \sum_{i=1}^{M} \text { Step }_{i},
$$

where Step $_{i}$ is the number of stopping steps in $i$-th test, $i=1,2, \cdots, M$, and $M$ is the total times of tests. 


$$
\text { Avg-Val }=\frac{1}{M} \sum_{i=1}^{M} \mathrm{MAPE}_{i},
$$

where $\mathrm{MAPE}_{i}$ is the MAPE when the algorithm stopping in $i$-th test.

$$
\begin{aligned}
\text { MAD } & =\frac{1}{n-1} \sum_{i=2}^{n}\left|x_{i}^{(0)}-\hat{x}_{i}^{(0)}\right| ; \\
\text { RMSE } & =\sqrt{\frac{1}{n-1} \sum_{i=2}^{n}\left(x_{i}^{(0)}-\hat{x}_{i}^{(0)}\right)^{2}} .
\end{aligned}
$$

To evaluate the performance of WOA, three classical intelligent heuristic algorithms, i.e. particle swarm optimization (PSO, with the inertia weight $=0.7298$ and $c_{1}=c_{2}=1.4962$ ), grey wolf optimizer (GWO), and genetic algorithm (GA, with crossover probability $=0.8$ and mutation probability $=0.1$ ), are considered when determining the evaluation parameters in $\operatorname{CFGM}(1, \mathrm{M})$ model, $\lambda, v_{3}, v_{4}, \cdots, v_{N}$. Therefore, four algorithms are tested with the MATLAB 2017a in Windows 10 (where CPU is Intel Core i7-6700 3.40 GHz and RAM is 16.0 GB).

The population sizes in these algorithms all are 50 and the maximum of iterations are 200. To save the computing time, these algorithms will be terminated if the variation of optimization objective keeps less than $10^{-7}$ for 6 iterations continuously. For each heuristic algorithm, six group data, divided from the modeling datasets, are adopt to establish the $\operatorname{CFGM}(1, N)$ model. To evaluate the convergence and convergence rate, this research lists two metrics in Table 4 after repeating these four algorithms 100 times. The one is the average step (Avg-Step) when the algorithms is stopped, the other is the average value (Avg-Val), namely, the average of MAPE of the CFGM $(1, N)$, at the termination. These results are detail in Table 4 . For improving the readability of this research, the best performance in each metrics are marked with the black bold font, and the suboptimal (just weaker than the best) performance are marked with the red bold font.

Table 4. The comparison about these four heuristic algorithms

\begin{tabular}{|c|c|c|c|c|c|c|c|c|}
\hline \multirow{2}{*}{ Data } & \multicolumn{2}{|c|}{ Models } & WOA-CFGM(1,N) & \multicolumn{2}{|c|}{ PSO-CFGM(1,N) } & \multicolumn{2}{c|}{ GWO-CFGM(1,N) } & \multicolumn{2}{c|}{ GA-CFGM(1,N) } \\
\cline { 2 - 9 } & Avg-Val & Avg-Step & Avg-Val & Avg-Step & Avg-Val & Avg-Step & Avg-Val & Avg-Step \\
\hline $2008-2012$ & $\mathbf{0 . 6 0 2 7 \%}$ & $\mathbf{2 3 . 3 7 3 2}$ & $\mathbf{0 . 6 0 2 7 \%}$ & 27.1292 & $\mathbf{0 . 6 0 2 7 \%}$ & 25.7115 & $0.6036 \%$ & $\mathbf{2 1 . 9 7 0 9}$ \\
\hline $2008-2013$ & $\mathbf{4 . 1 2 8 8} \%$ & $\mathbf{2 4 . 7 5 3 5}$ & $4.1290 \%$ & 34.6294 & $4.1289 \%$ & 28.9438 & $4.1292 \%$ & $\mathbf{2 2 . 3 4 1 2}$ \\
\hline $2008-2014$ & $\mathbf{5 . 2 7 6 0} \%$ & $\mathbf{2 3 . 6 0 8 3}$ & $5.2763 \%$ & 30.5269 & $\mathbf{5 . 2 7 6 0} \%$ & 27.5305 & $5.2763 \%$ & $\mathbf{1 8 . 2 4 5 9}$ \\
\hline $2008-2015$ & $\mathbf{4 . 8 4 2 9} \%$ & $\mathbf{2 6 . 3 5 2 1}$ & $\mathbf{4 . 8 4 2 9} \%$ & 33.7528 & $4.8431 \%$ & 28.1296 & $4.8437 \%$ & $\mathbf{1 9 . 5 6 7 0}$ \\
\hline $2008-2016$ & $\mathbf{4 . 4 9 2 0} \%$ & $\mathbf{2 3 . 8 4 4 1}$ & $4.4921 \%$ & 32.1146 & $4.4921 \%$ & 27.5544 & $4.4926 \%$ & $\mathbf{2 3 . 2 2 7 1}$ \\
\hline $2008-2017$ & $\mathbf{4 . 0 8 1 5} \%$ & $\mathbf{2 5 . 8 5 8 5}$ & $4.0823 \%$ & 30.0862 & $\mathbf{4 . 0 8 1 5} \%$ & 29.0756 & $4.0830 \%$ & $\mathbf{2 2 . 1 9 1 1}$ \\
\hline
\end{tabular}

Note: WOA-CFGM $(1, \mathrm{~N})$ means the $\operatorname{CFGM}(1, \mathrm{~N})$ using the WOA for parameter optimization, PSO-CF$\operatorname{GM}(1, \mathrm{~N})$ means the CFGM $(1, \mathrm{~N})$ using the PSO, GWO-CFGM $(1, \mathrm{~N})$ means the CFGM $(1, \mathrm{~N})$ using the GWO, and GA-CFGM $(1, N)$ means the $\operatorname{CFGM}(1, N)$ using the GA. 
In these four heuristic algorithms, GA seems always to have the best performance in the convergence (Avg-step), but it can't find the best value for $\operatorname{CFGM}(1, N)$ model, which means the GA has a high risk of premature in these dataset at the same stopped criteria error. Although just preforming suboptimal in Avg-step, WOA always gains a smallest MAPE at the termination, which means the WOA have a best performance on discouraging premature convergence. PSO and GWO also can find the best value for our model, but they both have a larger Avg-Step in this comparison, which means they may waste more time than GA even WOA. Thus, the WOA is recommended in $\operatorname{CFGM}(1, \mathrm{~N})$ model.

After comparing the performance of optimization algorithm in $\operatorname{CFGM}(1, \mathrm{~N})$ model, this research then evaluates the model interpretation three grey multivariate models, i.e. the proposed model, the classical GM(1,N) model (Zeng et al., 2016) and the $\mathrm{GM}(1, \mathrm{~N})$ with the interaction effect (abbreviated as $\operatorname{IEGM}(1, N)$, seeing in Wang (2017). For each model, six subsystems, divided from the modeling dataset, are used to construct these three kind of grey models. Thus, 18 grey multivariate models are established with the same research starting point of 2008. To understand and interpret models in a better way, the model performance evaluation metric is a widely used techniques. Then besides MAPE, mean absolute deviation (MAD) and root mean squared error (RMSE) both are taken as the metrics to evaluate the model performance.

Based on the three performance evaluation metrics in Table 5, the CFGM $(1, N)$ model is more reasonable for evaluating the coordination between China's S\&T and economy system than the classical GM(1,N) model and $\operatorname{IEGM}(1, \mathrm{~N})$ model. The MAPEs of the six sub-models are all approximately $5 \%$, and the values of MAE and RMSE are kept below 0.1 , suggesting that these six $\operatorname{CFGM}(1, N)$ models have a better performance in fitting. Namely, the unobserved errors of CFGM $(1, N)$ models are smaller than two other models. Thus, comparing with the classical GM(1,N) models and $\operatorname{IEGM}(1, N)$ models, all of them perform better in the interpretation. And it is necessary to consider the interaction effect quantitatively in modelling, which can make the following coordination evaluation in China's S\&T and economy system more trustworthy.

Table 5. Evaluation about the grey model interpretation

\begin{tabular}{|c|c|c|c|c|c|c|c|c|c|}
\hline \multirow{2}{*}{ Models } & \multicolumn{3}{|c|}{ CFGM(1,N) } & \multicolumn{3}{c|}{ GM(1,N) } & \multicolumn{3}{c|}{ IEGM(1,N) } \\
\cline { 2 - 10 } & MAPE & MAE & RSME & MAPE & MAE & RSME & MAPE & MAE & RSME \\
\hline $2008-2012$ & $\mathbf{0 . 6 0 \%}$ & $\mathbf{0 . 0 1 0 2}$ & $\mathbf{0 . 0 0 9 5}$ & $23.32 \%$ & 0.5017 & 0.4369 & $22.25 \%$ & 0.4746 & 0.4148 \\
\hline $2008-2013$ & $\mathbf{4 . 1 3 \%}$ & $\mathbf{0 . 0 7 8 0}$ & $\mathbf{0 . 0 7 4 2}$ & $18.86 \%$ & 0.4204 & 0.3688 & $16.81 \%$ & 0.3693 & 0.3228 \\
\hline $2008-2014$ & $\mathbf{5 . 2 8 \%}$ & $\mathbf{0 . 1 0 4 4}$ & $\mathbf{0 . 0 8 3 4}$ & $13.44 \%$ & 0.3194 & 0.2656 & $16.61 \%$ & 0.3951 & 0.3403 \\
\hline $2008-2015$ & $\mathbf{4 . 8 4 \%}$ & $\mathbf{0 . 1 0 5 9}$ & $\mathbf{0 . 0 7 1 8}$ & $12.45 \%$ & 0.3034 & 0.2532 & $19.46 \%$ & 0.4873 & 0.4297 \\
\hline $2008-2016$ & $\mathbf{4 . 4 9 \%}$ & $\mathbf{0 . 0 9 8 8}$ & $\mathbf{0 . 0 7 1 1}$ & $9.60 \%$ & 0.2375 & 0.2047 & $18.98 \%$ & 0.4995 & 0.4476 \\
\hline $2008-2017$ & $\mathbf{4 . 0 8 \%}$ & $\mathbf{0 . 0 9 3 2}$ & $\mathbf{0 . 0 6 7 9}$ & $5.42 \%$ & 0.1401 & 0.1149 & $19.16 \%$ & 0.5203 & 0.4715 \\
\hline
\end{tabular}




\subsection{Model interpretation}

Given that the CFGM $(1, N)$ model's performance was better, the parameter estimation results for this observation system, i.e. the development coordination coefficients $a, b_{2}, b_{3}$, the interaction coefficient $\lambda$ of interaction among factors, and the coordination degree parameters (dissipative structure comentropy $S(k)$ and comentropy change value $\Delta S(k)$ ) of the six subsystems are presented in Figure 4, Figure 5 and Table 6.

These coordination coefficients of the comprehensive capital $b_{2}$, reveal that the comprehensive capital investment always promotes company profits during 2008 2017. Figure 4 illustrates that all $b_{2}>0$ indicates that the comprehensive capital investment always keeps a positive influence on corporate profits, which is consistent with the previous research (Krammer, 2017; Fu \& Zheng, 2018). And the increasing of the comprehensive capital investment also ensures the rapid and sustainable growth of the corporate profits.

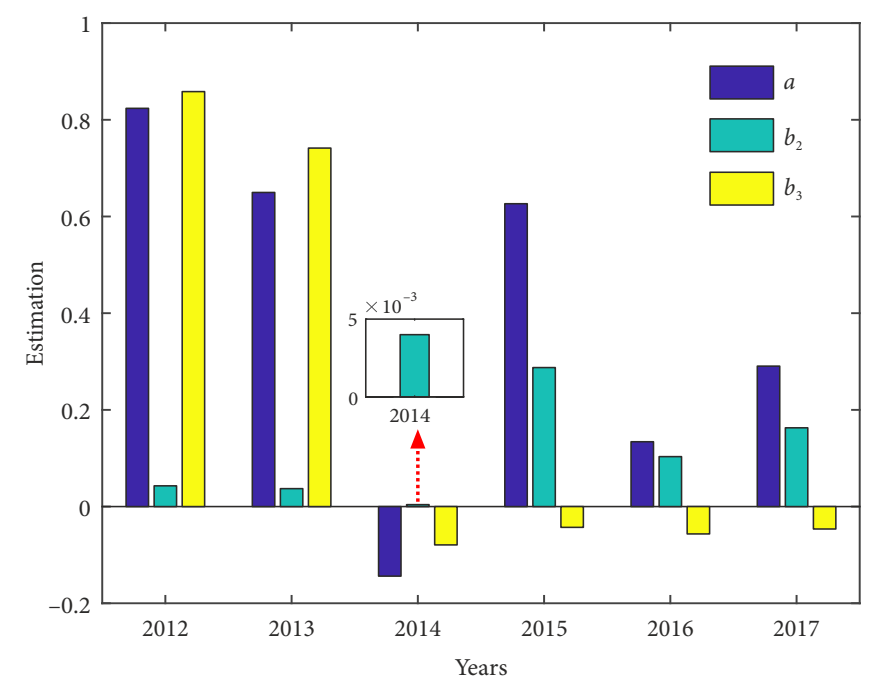

Figure 4. Description of model development coordination coefficient

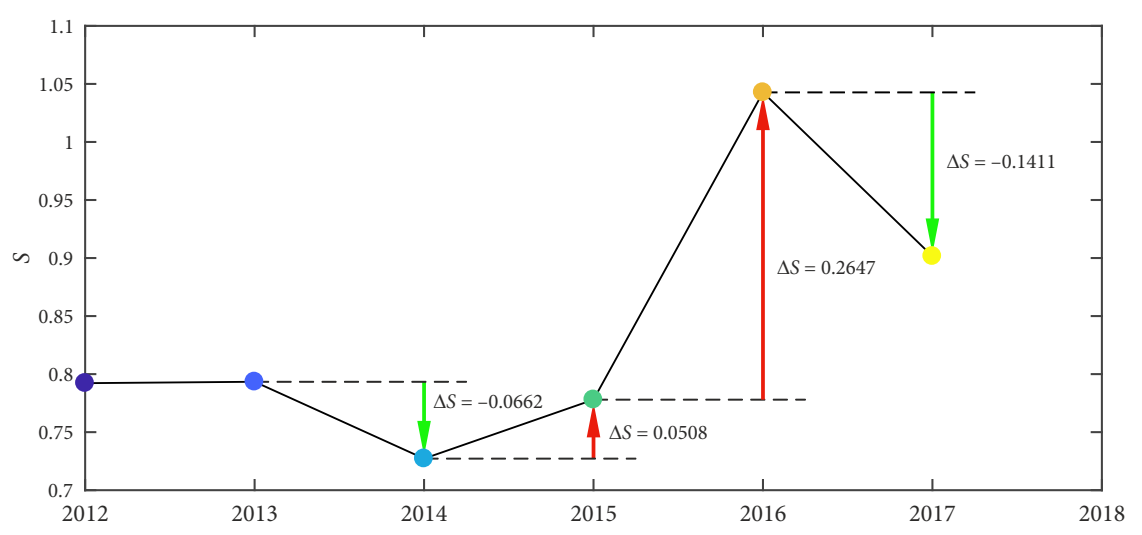

Figure 5. Grey information comentropy evaluation model 
Table 6. Results of CFGM(1,N) model

\begin{tabular}{|c|c|c|c|c|c|c|}
\hline \multirow{2}{*}{ Coefficients } & \multicolumn{3}{|c|}{ Development coordination } & Interaction & \multicolumn{2}{c|}{ Coordination degree } \\
\cline { 2 - 7 } & $a$ & $b_{2}$ & $b_{3}$ & 1 & $S(k)$ & $\Delta S(k)$ \\
\hline $2008-2012$ & 0.8237 & 0.0429 & 0.8583 & -1 & 0.7922 & 0 \\
\hline $2008-2013$ & 0.6497 & 0.0371 & 0.7415 & -1 & 0.7934 & 0.0013 \\
\hline $2008-2014$ & -0.1439 & 0.0040 & -0.0793 & -1 & 0.7272 & -0.0662 \\
\hline $2008-2015$ & 0.6264 & 0.2875 & -0.0430 & -1 & 0.7780 & 0.0507 \\
\hline $2008-2016$ & 0.1341 & 0.1033 & -0.0566 & -1 & 1.0427 & 0.2647 \\
\hline $2008-2017$ & 0.2905 & 0.1629 & -0.0464 & -1 & 0.9016 & -0.1411 \\
\hline
\end{tabular}

These development coefficients show that the Chinese technology and economic system is basically uncoordinated in self-development of the economic level. Figure 4 displays that except for 2014, $a>0$, indicating that the internal motivation of the self-development of the economic level is insufficient. The simple comprehensive human input has a restraining effect on the realization of final profit. The profit of an enterprise mainly depends on the continuous improvement of scientific and technological capabilities. As the development coefficient a continued to decline in 2012-2017, the incoordination degree of system gradually weakens, and the system's own mechanism is continuously improved. These results are consistent with increasing investment in science and technology development in China.

The coordination degrees from the comentropy suggest that China's technology and economic system is generally coordinated, and the coordination trend is consistent with the actual situation. Analyzing the comentropy of the coordination degree in Figure 5, $S(k)$, we can find all values of these six subsystem is basically between 0.7 and 1 . Namely, the coordination degree of China's technology and economic system always remains relatively stable over the past six years, and this phenomenon also suggests that the investment in S\&T always plays a positive role on the high-tech industry. The change about the comentropy reveals this phenomenon as well. Observing the change of comentropy in Figure 5, $\Delta S(k)$, we find it is negative during the period 2013-2014, which reveals that the order degree of China's $\mathrm{R} \& \mathrm{D}$ system gradually increases in the early stage of industrial development. $\Delta S(k)$ is positive during the period 2015-2016, with rapid improvement of scientific and technological capabilities. Therefore, the interaction effect between comprehensive capital investment and comprehensive human input in the S\&T input system has increasingly affected the hightech industry and has begun to form negative impacts and interference. $\Delta S(k)$ is negative in 2017. Thus, the coordination degree of China's R\&D system has gradually increased after a period of adjustment, thereby reaching more than 0.9 in 2017. Technology investment has also become a positive factor that promotes the development of China's high-tech industry. Therefore, the comentropy change result is consistent with the actual situation.

The coordination coefficients of the comprehensive human input, $b_{3}$, reveals that there may exists the personnel redundancy or lower per capita treatment in China's high-tech $\mathrm{R} \& \mathrm{D}$ investment. By observing coordination coefficient $b_{3}$ in Figure $4, b_{3}$ is initially positive and then becomes negative. Hence, the comprehensive manpower input has a positive and negative influence on the final profit in 2012-2013 and 2014-2017, respectively. With 2014 
as the dividing line, the full-time equivalent of $\mathrm{R} \& \mathrm{D}$ personnel factor dominates because of the comprehensive human resource input factors. Personnel investment after 2014 is also significantly higher than the three previous years. Hence, the phenomenon that there may exist the personnel redundancy or low per capita treatment occurred after 2014.

These interaction coefficients $(\lambda)$ suggest that there exists a strong negative cooperation between the comprehensive human input and the comprehensive capital investment. The quantitative observation about the interaction effect with fuzzy measure is our innovative exploration, and it's also the expansion from the classical GM(1,N) model. The interaction coefficients of these six subsystems are all $\mathbf{- 1}$, suggesting that there exists a strong and negative cooperation between $x_{2}^{(0)}$ and $x_{3}^{(0)}$. Namely, $x_{2}^{(0)}$ and $x_{3}^{(0)}$ are mutually inhibiting in the factors set. Although surprising, this finding can also be verified from Figure 4, where the comprehensive human input initially increased and then decreased with the continuous increase of comprehensive capital investment. And this finding also ensures the analysis about $b_{3}$ above.

These results are objective and credible, which could help identify contributors in China's technology and economic and understand the complicated coupling relationship, and then implement the sustainable development strategies to better balance economic growth and technology development. However, the assessment results are constrained by the characteristics of the research data. This is because the model is assigned a macroscopic coordination relationship and the heterogeneity in various microcosmic industries that cannot yet be implemented, although it has been used for a number of evaluations. Obviously, the evolution of the microcosmic coupling coordination degree is more useful for programming the development of a specific industrial. Consequently, further studies are essential to collect more detailed industries characteristic data and develop dynamic microcosmic coupling coordination degree in corresponding industries in the future.

\section{Conclusions and insights}

Aiming at the background of China's R\&D system coordination degree evaluation and considering the correlation, interaction, and small sample data characteristics among the factor sequences in the evaluation system, this study establishes a $\operatorname{CFGM}(1, \mathrm{~N})$ model to evaluate system coordination degree. The main innovations are explained as follows.

(1) Based on fuzzy integral theory, a novel grey multivariable $\operatorname{CFGM}(1, \mathrm{~N})$ evaluation model with fuzzy integral is established. The main form of the model, the parameter estimation method, and the whitening differential equation are solved. The subsequent model evaluation is also completed. The model can reflect the interactivity and improve the interpretability of the model.

(2) The parameters in the $\operatorname{CFGM}(1, N)$ model are optimized by WOA. The parameter optimization model is also designed. The fuzzy integral parameters in the model are solved by WOA. Technology can mine in-depth data information and complete the measurement of the interaction degree among factors and that of coordination coefficient. 
(3) Based on the real data of the Chinese R\&D system, an empirical analysis of the coordination degree assessment is conducted. Three types of parameters, which are model coordination coefficient, coordination degree, and comentropy change value, are proposed to comprehensively evaluate the coordination relationship between input in S\&T and output of high-tech. The research results reveal that comprehensive capital investment and human resource investment of high-tech industries have a mutual restraining effect for corporate profits. Increasing the investment of human resources may inhibit corporate profits, and the current high-tech industry R\&D investment system has human resources investing redundancy or low treatment of R\&D personnel. China's R\&D system is relatively coordinated, and the trend of coordination degree is consistent with the actual situation. Technology development and investment are becoming positive factors for promoting the rapid and sustainable development of China's high-tech industries.

\section{Acknowledgements}

This work is partially supported by the China Scholarship Council (CSC) (Grant No. 201906130025, 201906130049) and the National Natural Science Foundation of China (Grant No. 71871174).

\section{References}

Aggarwal, M. (2017). Learning of aggregation models in multi criteria decision making. KnowledgeBased Systems, 119, 1-9. https://doi.org/10.1016/j.knosys.2016.09.031

Block, J., Miller, D., Jaskiewicz, P., \& Spiegel, F. (2013). Economic and technological importance of innovations in large family and founder firms: An analysis of patent data. Family Business Review, 26(2), 180-199. https://doi.org/10.1177/0894486513477454

Chiang, J. H. (1999). Choquet fuzzy integral-based hierarchical networks for decision analysis. IEEE Transactions on Fuzzy Systems, 7(1), 63-71. https://doi.org/10.1109/91.746311

Coccia, M. (2014). Driving forces of technological change: The relation between population growth and technological innovation: Analysis of the optimal interaction across countries. Technological Forecasting and Social Change, 82, 52-65. https://doi.org/10.1016/j.techfore.2013.06.001

Deng, J. (2002). The basis of grey theory. Huazhong University of Science \& Technology Press.

Ding, S., Dang, Y., Xu, L., \& Wang, J. (2018). Multivariable grey forecasting model based on interaction effect and its application. Systems Engineering \& Electronics, 40(3), 595-602. https://doi.org/10.3969/j.issn.1001-506X.2018.03.17

Feng, Y., Zhou, M., Tian, G., Li, Z., Zhang, Z., Zhang, Q., \& Tan, J. (2018). Target disassembly sequencing and scheme evaluation for CNC machine tools using improved multiobjective ant colony algorithm and fuzzy integral. IEEE Transactions on Systems, Man, and Cybernetics: Systems, 49(12), 2438-2451. https://doi.org/10.1109/TSMC.2018.2847448

Fu, Z., \& Zheng, R. (2018). Time delay multivariable GM(1,N) coordination model and its application. Statistics \& Decision, 34(13), 77-80. https://doi.org/10.13546/j.cnki.tjyjc.2018.13.017

Grabisch, M. (1996). The application of fuzzy integrals in multicriteria decision making. European Journal of Operational Research, 89(3), 445-456. https://doi.org/10.1016/0377-2217(95)00176-X 
Hong, J. P. (2017). Causal relationship between ICT R\&D investment and economic growth in Korea. Technological Forecasting and Social Change, 116, 70-75. https://doi.org/10.1016/j.techfore.2016.11.005

$\mathrm{Hu}$, Y. C. (2008). A novel fuzzy classifier with Choquet integral-based grey relational analysis for pattern classification problems. Soft Computing, 12(6), 523-533. https://doi.org/10.1007/s00500-007-0224-5

Ishida, H. (2015). The effect of ICT development on economic growth and energy consumption in Japan. Telematics and Informatics, 32(1), 79-88. https://doi.org/10.1016/j.tele.2014.04.003

Jiang, Y. (2011). Construction of forecasting and optimizing control model for coordinated development of regional science and technology systems. Statistics \& Decision, 6, 60-62. https://doi.org/10.13546/j.cnki.tjyjc.2011.06.006

Kadaifci, C., Asan, U., \& Bozdag, E. (2020). A new 2-additive Choquet integral based approach to qualitative cross-impact analysis considering interaction effects. Technological Forecasting and Social Change, 158, 120131. https://doi.org/10.1016/j.techfore.2020.120131

Khan, M. S. A., \& Abdullah, S. (2018). Interval - valued Pythagorean fuzzy GRA method for multipleattribute decision making with incomplete weight information. International Journal of Intelligent Systems, 33(8), 1689-1716. https://doi.org/10.1002/int.21992

Krammer, S. M. (2017). Science, technology and innovation for economic competitiveness: The role of smart specialization in less-developed countries. Technological Forecasting \& Social Change, 123, 95-107. https://doi.org/10.1016/j.techfore.2017.06.028

Marichal, J. L. (2004). Tolerant or intolerant character of interacting criteria in aggregation by the Choquet integral. European Journal of Operational Research, 155(3), 771-791. https://doi.org/10.1016/S0377-2217(02)00885-8

Meng, F., Zhang, Q., \& Zhan, J. (2015). The interval-valued intuitionistic fuzzy geometric Choquet aggregation operator based on the generalized Banzhaf index and 2-additive measure. Technological and Economic Development of Economy, 21(2), 186-215. https://doi.org/10.3846/20294913.2014.946983

Mirjalili, S., \& Lewis, A. (2016). The whale optimization algorithm. Advances in Engineering Software, 95, 51-67. https://doi.org/10.1016/j.advengsoft.2016.01.008

National Bureau of Statistics of China. (2020). Retrieved October 26, 2020, from https://data.stats.gov.cn

Neokosmidis, I., Avaritsiotis, N., \& Ventoura, Z. (2013). Modeling gender evolution and gap in science and technology using ecological dynamics. Expert Systems with Applications, 40(9), 3481-3490. https://doi.org/10.1016/j.eswa.2012.12.056

Pradhan, R. P., Arvin, M. B., Norman, N. R., \& Bele, S. K. (2014). Economic growth and the development of telecommunications infrastructure in the G-20 countries: A panel-VAR approach. Telecommunications Policy, 38(7), 634-649. https://doi.org/10.1016/j.telpol.2014.03.001

Qu, G., Qu, W., Zhang, Z., \& Wang, J. (2017). Choquet integral correlation coefficient of intuitionistic fuzzy sets and its applications. Journal of Intelligent \& Fuzzy Systems, 33(1), 543-553. https://doi.org/10.3233/JIFS-162131

Sugeno, M. (1974). Theory of fuzzy integral and its applications. Tokyo Institute of Technology.

Sun, J. (1996). Research of that science and technology economy society coordination development model. Chinese Journal of Management Science, 2, 13-18.

Takahagi, E. (2008). A fuzzy measure identification method by diamond pairwise comparisons and $\Phi \_s$ transformation. Fuzzy Optimization and Decision Making, 7(3), 219-232. https://doi.org/10.1007/s10700-008-9032-3

Tian, G., Hao, N., Zhou, M., Pedrycz, W., Zhang, C., Ma, F., \& Li, Z. (2019). Fuzzy grey choquet integral for evaluation of multicriteria decision making problems with interactive and qualitative indices. IEEE Transactions on Systems, Man, and Cybernetics: Systems, 1-14. https://doi.org/10.1109/TSMC.2019.2906635 
Wang, Y., \& Xu, Q. (2009). Comparison analysis on the coordination degree between science \& technology and economy in different regions in China. Science \& Technology Progress and Policy, 26(20), 29-31.

Wang, Z. (2017). Multivariable GM(1,N) model with interaction effects. Control and Decision, 32(3), $515-520$.

Wang, Z. X., He, L. Y., \& Li, D. D. (2019). Assessment of the degree of order in the organisational structure of electricity regulatory institution in China based on Shannon entropy. Energy Policy, 132, 429-439. https://doi.org/10.1016/j.enpol.2019.06.002

Xiao, Q., Gao, M., Xiao, X., \& Goh, M. (2020c). A novel grey Riccati-Bernoulli model and its application for the clean energy consumption prediction. Engineering Applications of Artificial Intelligence, 95, 103863. https://doi.org/10.1016/j.engappai.2020.103863

Xiao, Q., Shan, M., Gao, M., \& Xiao, X. (2020a). Grey information coverage interaction relational decision making and its application. Journal of Systems Engineering and Electronics, 31(2), 359-369. https://doi.org/10.23919/JSEE.2020.000013

Xiao, Q., Shan, M., Gao, M., Xiao, X., \& Goh, M. (2020b). Parameter optimization for nonlinear grey Bernoulli model on biomass energy consumption prediction. Applied Soft Computing, 95, 106538. https://doi.org/10.1016/j.asoc.2020.106538

Xiao, X., \& Duan, H. (2020). A new grey model for traffic flow mechanics. Engineering Applications of Artificial Intelligence, 88, 103350. https://doi.org/10.1016/j. engappai.2019.103350

Xiao, X., Duan, H., \& Wen, J. (2020d). A novel car-following inertia grey model and its application in forecasting short-term traffic flow. Applied Mathematical Modelling, 87, 546-570. https://doi.org/10.1016/j.apm.2020.06.020

Yuan, Y., Yang, J., Li, Y., \& Li, W. (2020). Necessary conditions for coordination of dual-channel closedloop supply chain. Technological Forecasting and Social Change, 151, 119823. https://doi.org/10.1016/j.techfore.2019.119823

Zeng, B., Luo, C., Liu, S., Bai, Y., \& Li, C. (2016). Development of an optimization method for the GM(1, N) model. Engineering Applications of Artificial Intelligence, 55, 353-362. https://doi.org/10.1016/j.engappai.2016.08.007

Zhang, W., Ju, Y., \& Liu, X. (2017). Multiple criteria decision analysis based on Shapley fuzzy measures and interval valued hesitant fuzzy linguistic numbers. Computers \& Industrial Engineering, 105, 28-38. https://doi.org/10.1016/j.cie.2016.12.046

Zhang, Y. (2014). A study of dynamic relationship between science \& technology investment and economic growth in China. Science Research Management, 35(9), 58-68.

https://doi.org/10.19571/j.cnki.1000-2995.2014.09.008 